\title{
Indentation instrumentée multi-échelles appliquée à l'étude des matériaux massifs métalliques
}

\author{
Stephania Kossman ${ }^{1,2, a}$, Didier Chicot ${ }^{1}$ et Alain Iost $^{2}$
}

Reçu le 18 novembre 2016, accepté le 6 février 2017

Résumé - L'essai d'indentation instrumentée est une technique très utilisée car elle permet d'estimer les propriétés mécaniques des matériaux comme la dureté et le module de Young. Cette étude sur l'indentation multi-échelles (de $5 \mathrm{mN}$ à $2 \mathrm{kN}$ ) a été réalisée sur des matériaux métalliques massifs (acier, acier inoxydable et aluminium) avec trois instruments : nano, micro et macro indenteurs, et différents types d'essais : classique, CSM et multicyclique. Nous avons constaté que, pour estimer les propriétés mécaniques, il est nécessaire de prendre en compte la correction du défaut de pointe et aussi de la complaisance du bâti quelle que soit l'échelle de mesure, et donc quelle que soit la conception de l'instrument pour la mesure du déplacement. Dans ces conditions, en considérant ces corrections, le module de Young reste quasi constant dans tout le domaine de force étudié $(<12 \%)$, et est donc à ce titre une propriété intrinsèque. Par contre, la dureté décroit lorsque la charge augmente, ce qui est classiquement attribué à l'effet de taille en indentation, mais qui peut aussi être imputé au mode de déformation autour de l'empreinte, au bourrelet prédominant (pile-up) pour les échantillons en acier et aluminium, et à la déflection des faces (sink-in) pour l'acier inoxydable.

Mots clés : Indentation multi-échelles / module de Young / dureté / modes de déformation

\begin{abstract}
Instrumented multiscale indentation applied to the study of bulk metallic materials. The instrumented indentation test (ITT) is a largely used technique, because it allows to estimate the mechanical properties of materials, as hardness and Young's modulus. This study about multi-scale ITT (from $5 \mathrm{mN}$ to $2 \mathrm{kN}$ ) was performed on bulk metallic materials (steel, stainless steel and aluminum) using three instruments: nano, micro and macro indenter, and different kinds of test: standard, CSM and multicycle. We have found that for the estimation of the mechanical properties, it is necessary to consider the correction of the frame compliance and tip defect, for all the scales of measurement and instruments. In these conditions, considering these corrections, the Young's modulus is almost constant among all the range of studied loads $(<12 \%)$, being an intrinsic property. On the contrary, the hardness decreases while increasing the load, which is typically attributed to the indentation size effect, but also it can be related to the deformation around the imprint, pile-up for the samples of steel and aluminum, and sink-in for the stainless steel.
\end{abstract}

Key words: Multiscale indentation / Young's modulus / hardness / surface deformation mode

\section{Introduction}

L'essai d'indentation instrumentée a été largement utilisé et étudié en raison de la facilité qu'il procure pour estimer les propriétés mécaniques des matériaux à partir de la courbe force-déplacement sur de petits volumes de matière $[1,2]$. Les principales propriétés qu'il permet d'obtenir sont le module de Young et la dureté, mais le coefficient d'écrouissage, la limite d'élasticité [3-5] et la ténacité à la rupture $[6,7]$ peuvent également être calculés à partir des courbes force-déplacement enregistrées.

Cette technique permet d'étudier les propriétés mécaniques des matériaux à différentes échelles. Un matériau homogène peut être testé indifféremment en nano, micro ou macro indentation; au contraire, pour des matériaux hétérogènes, la réponse à l'indentation

\footnotetext{
a Auteur de correspondance : stephaniakossman@gmail.com

1 Université de Lille, FRE 3723 - LML - Laboratoire de Mécanique de Lille, 59000 Lille, France

2 Arts et Métiers ParisTech, MSMP, 59800 Lille, France
} 


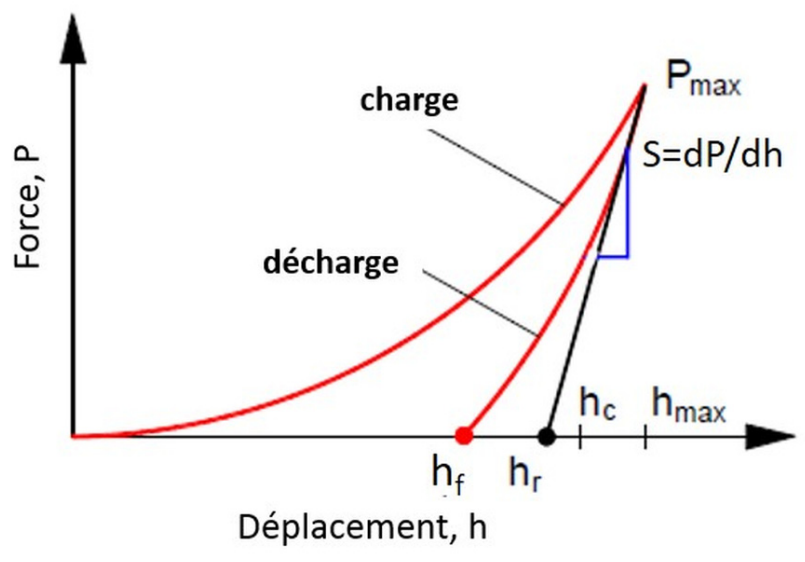

Fig. 1. Courbe force-déplacement d'un essai d'indentation instrumenté classique. $P_{\max }$ : force maximale, $h_{\max }=h_{m}$ : déplacement maximum, $h_{f}$ : profondeur résiduelle, $h_{r}$ : profondeur plastique, $S$ : raideur, et $h_{c}$ : profondeur de contact.

Fig. 1. Load-displacement curve from standard instrumented indentation test. $P_{\max }:$ maximal load, $h_{\max }=h_{m}:$ maximum displacement, $h_{f}$ : residual depth, $h_{r}:$ plastic depth, $S$ : contact stiffness, $h_{c}$ : contact depth.

dépendra du volume plastifié sous l'indenteur [8]. La norme ISO 14577 établi trois gammes d'application de l'essai en fonction de la profondeur indentée $(h)$ et de la force appliquée sur l'indenteur $(P)$ : nano $(h \leq 0,2 \mu \mathrm{m})$, micro $(2 N>P, h>0,2 \mu \mathrm{m})$ et macro $(2 N \leq P \leq$ $30 \mathrm{kN}$ ). L'analyse des données pour obtenir les propriétés mécaniques est similaire pour les trois échelles, la principale différence résidant dans le choix de la fonction utilisée pour estimer l'aire de contact.

La Figure 1 représente une courbe force-déplacement d'un essai d'indentation instrumentée classique, où sont identifiés les paramètres importants pour l'analyse.

La courbe de décharge est souvent supposée purement élastique et représentée par une relation puissance (Eq. (1)) [9],

$$
P=B\left(h-h_{f}\right)^{m} .
$$

Le module d'élasticité réduit $E_{R}$ du matériau (Eq. (2)) se détermine à partir de la raideur $S(\mathrm{~d} P / \mathrm{d} h)$ calculée à la profondeur maximale sur la courbe à la décharge, et de l'aire de contact $A_{c}$ :

$$
E_{R}=\frac{S \sqrt{\pi}}{2 \beta \gamma \sqrt{A}_{c}}
$$

avec $\beta$ un facteur relatif à la géométrie de la pointe (généralement 1,034 pour l'indenteur Berkovich et 1,012 pour l'indenteur Vickers), $\gamma$ voisin de 1,07 pour les matériaux métalliques, un facteur tenant compte des coefficients de Poisson et du déplacement radial [10].

$E_{R}$ est lié aux modules de Young et aux coefficients de Poisson de l'indenteur $\left(E_{i}, \nu_{i}\right)$ et du matériau $(E, \nu)$ par la relation :

$$
\frac{1}{E_{R}}=\frac{1-\nu_{i}^{2}}{E_{i}}+\frac{1-\nu^{2}}{E}
$$

où $E_{i}=1140 \mathrm{GPa} \mathrm{GPa}$ et $\nu_{i}=0,07$ pour un indenteur en diamant.

Pour un indenteur parfait, la dureté (HIT) est définie comme le rapport de la force maximale sur l'aire de contact projetée $\left(24,5 h_{c}^{2}\right)$, ou le rapport de la force maximale sur l'aire de contact réelle $\left(26,43 h_{\max }^{2}\right)$ pour la dureté Martens (HM) :

$$
H I T=\frac{P_{\max }}{24,5 h_{c}^{2}} \quad H M=\frac{P_{\max }}{26,43 h_{\max }^{2}} .
$$

Pour tenir compte des défauts de pointe, les fonctions d'aire développées pour le calcul de l'aire de contact projetée prennent des formes plus ou moins complexes (Eqs. (5)-(8)). Pour corriger l'aire de contact réelle, les fonctions d'aire utilisées sont les mêmes, sauf que la profondeur maximale $h_{\max }$ est substituée à la profondeur de contact $h_{c}$ et le coefficient 24,5 est remplacé par 26,43 excepté pour l'équation (5).

La fonction d'aire est une quantité critique à calculer, particulièrement en nano et microindentation avec les indenteurs pyramidaux où le défaut de pointe est important par rapport aux profondeurs de pénétration. En nanoindentation la fonction d'aire proposée par Oliver et Pharr [9] est la plus utilisée (Eq. (5)), pour laquelle les coefficients, $C_{n}$, sont les paramètres d'ajustement aux moindres carrés de la courbe obtenue à partir d'essais CSM (Continuous Stiffness Measurement) sur un matériau de propriétés connues, généralement la silice fondue,

$$
A_{c}=24,5 h_{c}^{2}+C_{1} h_{c}^{1}+C_{2} h_{c}^{1 / 2}+C_{3} h_{c}^{1 / 4}+\ldots+C_{8} h_{c}^{1 / 128}
$$

La relation développée par Chicot et al. [11] (Eq. (6)) permet également d'estimer l'aire de contact pour des profondeurs très faibles $(<200 \mathrm{~nm})$ dans le cas où la méthode CSM n'est pas disponible sur l'instrument utilisé. Le défaut de pointe, $h_{b}$, peut être soit déterminé par calibration sur un matériau connu, soit estimé par Microscopie Electronique à Balayage (MEB).

$$
A_{c}=24,5\left(h_{c}+h_{b}\left(1-\exp \left(-\frac{2 h_{c}}{h_{b}}\right)\right)^{3 / 2}\right)^{2} .
$$

L'équation 6 est aussi valable pour des profondeurs plus élevées (gamme de la microindentation) et donne des résultats comparables à ceux estimés par la relation de Troyon et al. [12] (Eq. (7)), qui revient à négliger le terme exponentiel de l'équation (6).

$$
A_{c}=24,5\left(h_{c}+h_{b}\right)^{2} .
$$

Pour la macroindentation, l'aire de contact (Eq. (8)) est généralement considérée comme ayant la forme d'une pyramide parfaite car les déplacements $(>5 \mu \mathrm{m})$ sont très importants par rapport au défaut de pointe possible de l'indenteur.

$$
A_{c}=24,5 h_{c}^{2}
$$

La profondeur de contact utilisée, $h_{c}$, change selon le mode de déformation prédominant dans le matériau. La 
méthode d'Oliver et Pharr [13] est utilisée quand le matériau présente une déflection des faces de l'empreinte de dureté (sink-in) (Eq. (9)), et la méthode de Loubet et al. $[14,15]$ quand le bourrelet (pile-up) est prédominant (Eq. (10)).

$$
\begin{aligned}
& h_{c_{\_} \text {sink-in }}=h_{\max }-0,75 \frac{P_{\max }}{S} \\
& h_{c_{-p} i l e-u p}=1,2\left(h_{\max }-\frac{P_{\max }}{S}\right)
\end{aligned}
$$

Un autre facteur important dans l'analyse de la courbe d'indentation est la détermination de la complaisance du bâti, $C_{f}$, liée à la déformation de l'instrument. L'inverse de la raideur, mesurée par la pente à la décharge, correspond à la complaisance totale $C_{T}$ du système décrit par l'équation (11) comme étant la somme de la complaisance du bâti ou de la machine, $C_{f}$, et de la complaisance du contact lié au matériau, $C_{s}$, décrit par le deuxième terme. Souvent, la correction de la complaisance de la machine est incluse dans le logiciel de l'instrument. Cependant, plusieurs éléments dans le système peuvent influer sur sa valeur, et la calibration doit être vérifiée dans toute la gamme de charges à utiliser. La norme ISO 14577-4 décrit dans son annexe A différents processus pour réaliser la calibration. Dans notre étude, nous avons utilisé la méthode 2 , avec comme hypothèses un module de Young constant, une fonction d'aire connue et une dureté variable avec la profondeur indentée. Un exemple de la calibration est montré sur la Figure 2.

$$
C_{T}=C_{f}+\frac{\sqrt{\pi}}{2} \frac{1}{\beta \gamma E_{R} \sqrt{A}_{c}} .
$$

Par la suite, les relations montrées précédemment sont utilisées pour estimer le module de Young et la dureté. Nous allons comparer et analyser les résultats d'indentation instrumentée dans une approche multi-échelles, où nous travaillons avec trois instruments à différentes échelles de force $(\mathrm{du} \mathrm{mN}$ au $\mathrm{kN}$ ) et de déplacement (du $\mathrm{nm}$ au $\mu \mathrm{m}$ ). L'information donnée à chaque échelle peut varier significativement pour les matériaux hétérogènes qui peuvent montrer des propriétés variables en nano, micro et macroindentation à cause de l'hétérogénéité de la structure et la présence de plusieurs phases.

\section{Méthodologie expérimentale}

Pour la caractérisation multi-échelles, quatre matériaux ont été utilisés, deux cales étalon en acier (39HRC et $63,4 \mathrm{HRC})$, un alliage d'aluminium $(\mathrm{Al})$ et un acier inoxydable (SS304).

Les matériaux ont été usinés pour avoir des surfaces planes, polies avec des papiers abrasifs de grades 80 jusqu'à 4000, puis avec une suspension d'alumine pour obtenir un polissage miroir. Entre chaque étape, les échantillons sont rincés et tournés de 45 degrés pour enlever les rayures précédentes et réduire l'effet d'écrouissage. Pour les échantillons en acier, l'éthanol a été utilisé

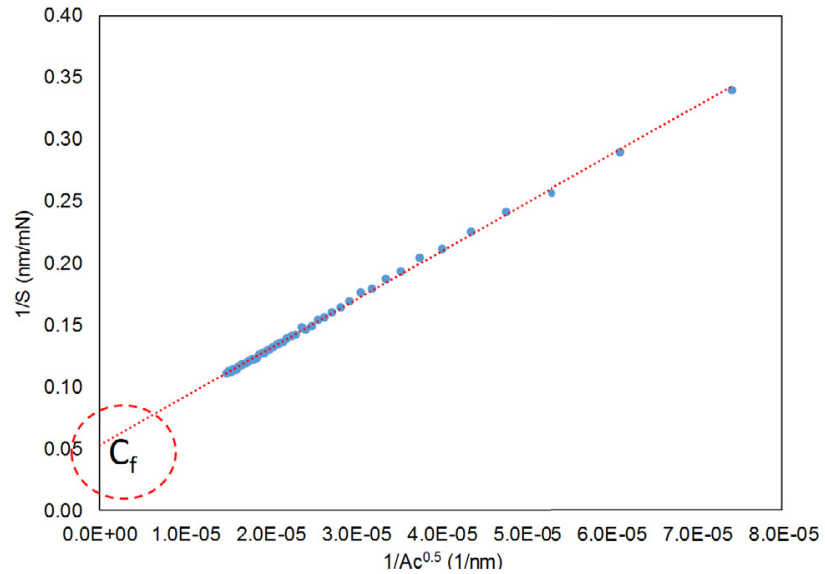

Fig. 2. Complaisance total mesurée à partir de l'inverse de la pente à la décharge en fonction de l'inverse de la racine carré de l'aire de contact supposée connue. L'interception de cette droite avec l'axe des ordonnées correspond à la valeur de la complaisance de la machine $C_{f}$.

Fig. 2. Total compliance measured from the inverse slope of unloading curve as a function of the inverse square root of the contact area assumed as a known relation. The intercept of the straight line with the ordinate axis represents the frame compliance, $C_{f}$.

comme lubrifiant pour éviter la corrosion; pour les autres échantillons, aluminium et acier inoxydable, de l'eau a été utilisée.

Les conditions expérimentales pour chaque instrument sont présentées sur les Tableaux 1 et 2 . Au moins 10 essais par condition ont été réalisés.

Il est important de mentionner que les trois instruments permettent d'utiliser des d'échantillons de tailles différentes. En nanoindentation celle-ci est limitée à $30 \mathrm{~mm}$ de diamètre et $10 \mathrm{~mm}$ de hauteur, mais il est possible d'analyser des échantillons de très faible épaisseur. En microindentation, l'échantillon doit être de préférence rectangulaire ou carré pour une meilleure fixation dans l'étau, la taille est approximativement de $30 \mathrm{~mm} \times 30 \mathrm{~mm}$ et la hauteur jusqu'à environ $30 \mathrm{~mm}$. En revanche, dans la machine de macroindentation, les échantillons peuvent être de taille supérieure à $15 \times 10 \mathrm{~cm}$, avec une hauteur minimale de $10 \mathrm{~mm}$.

Pour notre étude, nous avons utilisé des échantillons compatibles avec l'essai de nanoindentation pour pouvoir tester les mêmes échantillons avec les trois instruments.

\section{Résultats et discussion}

\section{1 Évaluation des modes de déformation}

Pour sélectionner le mode de déformation de chaque matériau, nous avons utilisé le rapport $h_{f} / h_{m}$ et la profilométrie optique. La Figure 3 montre les valeurs moyennes obtenues à partir des courbes force-déplacement pour chaque instrument et celles mesurées par profilométrie optique des empreintes de macroindentation. 
Tableau 1. Types d'essais et conditions en nano, micro et macroindentation.

Table 1. Types of tests and conditions in nano, micro and macroindentation.

\begin{tabular}{|c|c|}
\hline Machine & Type d'essai \\
\hline Nano Nano indenter XP, MTS & $\begin{array}{l}\text { Classique : temps constante ( } 30 \mathrm{~s} \text { charge/décharge) ; temps d'at- } \\
\text { tente, } 15 \mathrm{~s} \text {. CSM : vitesse de déformation, } 0,05 \mathrm{~s}^{-1} \text {; déplacement } \\
\text { harmonique, } 2 \mathrm{~nm} \text {; fréquence, } 45 \mathrm{~Hz}\end{array}$ \\
\hline Micro CSM2-107, CSM Instruments & $\begin{array}{l}\text { Multicyclique : } 50 \text { cycles, temps constant }(30 \mathrm{~s} \\
\text { charge/décharge); temps d'attente à } P_{\max }, 15 \mathrm{~s} ; \text { temps } \\
\text { d'attente entre cycles, } 15 \mathrm{~s} ; \text { décharge jusqu'à } 20 \% \text { de } P_{\max } \text { à } \\
\text { chaque cycle }\end{array}$ \\
\hline Macro ZHU 2.5, Zwick & $\begin{array}{l}\text { Classique : temps constante (30 s charge/décharge); temps d'at- } \\
\text { tente, } 15 \mathrm{~s} .\end{array}$ \\
\hline
\end{tabular}

Tableau 2. Conditions d'essais en nano, micro et macroindentation.

Table 2. Test parameters in nano, micro and macroindentation.

\begin{tabular}{cccc}
\hline Conditions & Nano & Micro & Macro \\
\hline Type d'indenteur & Berkovich & Berkovich & Vickers \\
\hline Gamme de charge & $1-650 \mathrm{mN}$ & $100 \mathrm{mN}-20 \mathrm{~N}$ & $5-2000 \mathrm{~N}$ \\
\hline $\begin{array}{c}\text { Méthode de } \\
\text { fixation échantillon }\end{array}$ & $\begin{array}{c}\text { Colle à chaud + support } \\
\text { porte-échantillon vissé }\end{array}$ & Étau & $\begin{array}{c}\text { Pâte à modeler autour } \\
\text { de l'échantillon posé sur la table }\end{array}$ \\
\hline $\begin{array}{c}\text { Détermination } \\
\text { défaut de pointe }\end{array}$ & $\begin{array}{c}\text { Calibration avec la silice } \\
\text { fondue, obtention des coefficients } \\
\text { pour la fonction d'aire (Eq. (5)) }\end{array}$ & $\begin{array}{c}\text { Mesure du défaut } \\
\text { de pointe par MEB (Eq. (6)) }\end{array}$ & $\begin{array}{c}\text { Hypothèse pyramide } \\
\text { parfaite }\end{array}$ \\
\hline
\end{tabular}

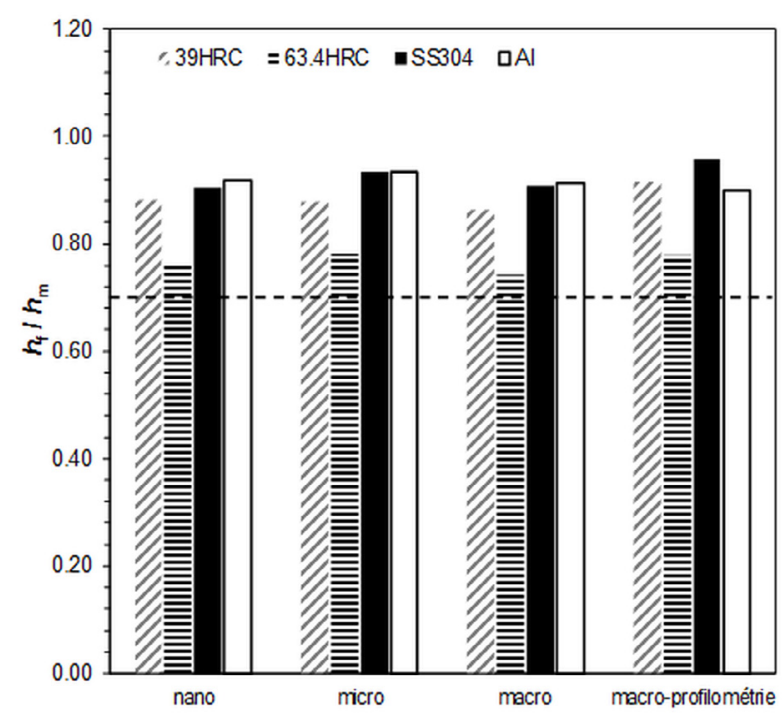

Fig. 3. Evaluation du rapport $h_{f} / h_{m}$ pour prédire le mode de déformation, les valeurs montrées représentent la moyenne du rapport obtenue à chaque échelle par l'analyse de courbes et aussi par profilométrie optique en empreintes de macroindentation.

Fig. 3. Evaluation of ratio $h_{f} / h_{m}$ to predict the deformation mode, the represented values correspond the average at each scale recorded on the indentation curves or by optical profilometry in macroindentation indents.

Les rapports $h_{f} / h_{m}$ restent constants dans toutes les gammes de charges et sont aussi comparables avec les valeurs obtenues par profilométrie optique. Tous les valeurs de $h_{f} / h_{m}$ sont supérieures à 0,7 , pour lesquelles le mode de déformation prédominant devrait être le bourrelet $[13,16]$.

Cependant, la relation relative à la présence du bourrelet utilisée dans le cas de l'acier inoxydable conduit à une sous-estimation importante du module de Young avec les trois instruments (approximativement $160 \mathrm{GPa}$ ) qui est une valeur très faible par rapport à la valeur théorique du module de Young (190-210 GPa) [17]. En fait, des études précédentes ont montré que la relation de Oliver et Pharr qui donne la profondeur de contact (Eq. (9)), reste pertinente dans le cas des matériaux qui possèdent une grande capacité d'écrouissage comme l'acier inoxydable. Dans ce cas, seul un petit bourrelet se forme mais le durcissement autour de l'empreinte restreint le mouvement de matière vers le haut, et la zone plastique va se développer plutôt en profondeur qu'en surface $[13,16]$. La Figure 4 présente un exemple de relevé topographique effectué sur ce matériau. Pour les autres trois matériaux (39HRC, 63.4HRC et $\mathrm{Al}$ ), la relation, considérant comme mode de déformation prédominant le bourrelet, utilisée pour estimer la profondeur de contact (Eq. (10)) permet une estimation correcte du module de Young .

\subsection{Variation du module de Young et de la dureté en fonction de la charge}

Les Equations (2) et (3) permettent d'estimer le module de Young à partir de la représentation de $1 / S$ en fonction de $1 / A_{c}^{0,5}$ (Fig. 5). Pour cette estimation on suppose que le module est constant avec la force d'essai et que la fonction d'aire est connue.

Les résultats précédents correspondent à la formation d'un bourrelet comme mode de déformation principal pour les échantillons d'acier et d'aluminium, et une 

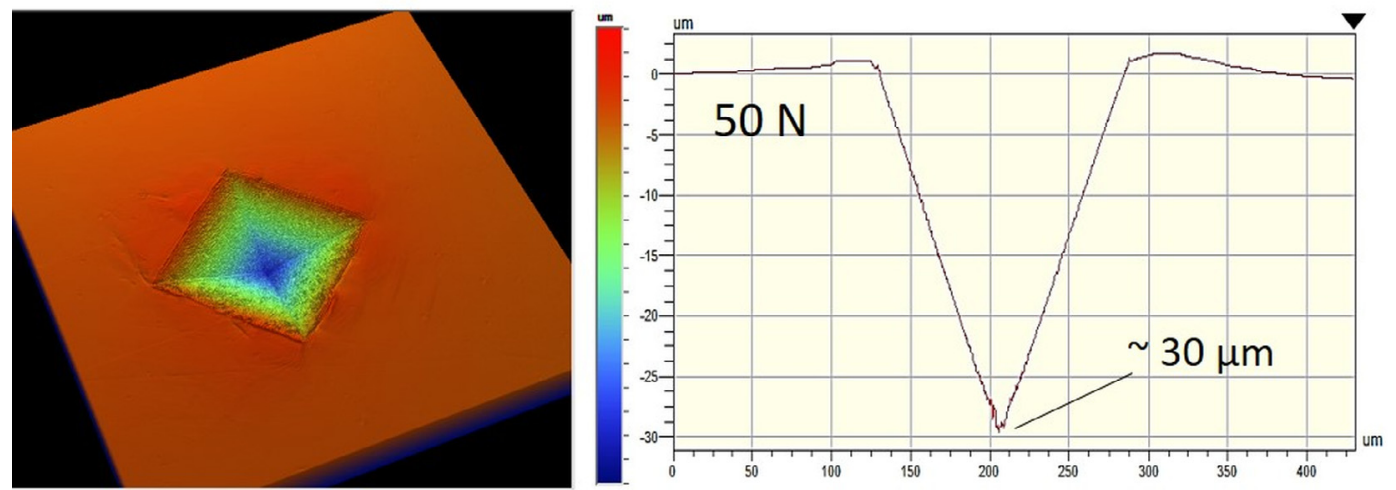

Fig. 4. Profilométrie optique d'une empreinte obtenue par macroindentation à $50 \mathrm{~N}$ sur l'acier inoxydable SS304.

Fig. 4. Optical profilometry of an imprint obtained by macroindentation at $50 \mathrm{~N}$ on a stainless steel SS304.

Tableau 3. Module de Young (GPa) estimé à partir de la pente $1 / \mathrm{S}$ en fonction de $1 / A_{c}^{0,5}$

Table 3. Young's modulus ( $G P a$ ) estimated from the slope of $1 / S$ as a function of $1 / A_{c}^{0.5}$.

\begin{tabular}{ccccc}
\hline Échelle & 39HRC & $63.4 \mathrm{HRC}$ & Aluminium & SS304 \\
\hline Nano Loubet & 197 & 198 & 67 & 165 \\
Nano O\&P & 237 & 226 & 79 & 197 \\
Micro Loubet & 192 & 197 & 67 & 157 \\
Micro O\&P & 230 & 229 & 80 & 190 \\
Macro Loubet & 170 & 189 & 65 & 150 \\
Macro O\&P & 204 & 218 & 77 & 182 \\
Valeur théorique & $190-210[18]$ & $190-210[18]$ & $65-100[19]$ & $190-210[17]$ \\
\hline
\end{tabular}

déflection des faces pour l'échantillon en acier inoxydable. Les valeurs du module de Young sont surestimées pour les échantillons d'acier et aluminium si la relation d'Oliver et Pharr est utilisée pour obtenir la profondeur de contact (la pente $1 / \mathrm{S}$ vs. $1 / A_{c}^{0,5}$ diminue), les valeurs sont présentées sur le Tableau 3. La variation entre les deux relations d'Oliver et Pharr et celle de Loubet et al. (Eqs. (9) et (10)) pour estimer la profondeur de contact donne une différence de $20 \%$ sur le module de Young . Par ailleurs, le module de l'acier inoxydable SS304, si la relation de Loubet et al. est utilisée, diminue $20 \%$ (160 GPa), lequel est inférieur à la valeur théorique.

Sur cette Figure 5 nous constatons, pour les quatre matériaux étudiés, que les modules de Young estimés avec les trois instruments sont comparables avec une variation entre $5 \%$ et $12 \%$. En macroindentation le module diminue légèrement de manière systématique, plus particulièrement pour le matériau 39HRC. Les différences constatées sont dans le domaine d'incertitude pour l'essai d'indentation qui peut être d'après la littérature autour de $10 \%$ [20,21].

Le petit changement de pente en macroindentation peut être lié aux relations utilisées pour décrire la profondeur de contact (Eqs. (9)-(10)) et la fonction d'aire (Eq. (8)) qui implique que la pyramide est parfaite; ce qui est contredit par quelques études qui mettent en évidence l'influence de l'existence d'un défaut de pointe même à l'échelle macrométrique [21,22].

Les Figures 6 et 7 présentent la variation du module de Young et de la dureté avec la charge et le déplacement pour les quatre matériaux étudiés. Les valeurs moyennes du module de Young sont comparables avec celles trouvées sur la Figure 5. En général, les propriétés présentent une continuité entre les différentes échelles de mesure, puisque le module est une propriété intrinsèque du matériau, donc indépendant de la force. Cependant, l'exemple du matériau 39HRC montre une différence plus importante (12\%) entre les essais de nano ou microindentation avec ceux de macroindentation. Cette différence est probablement liée à des erreurs expérimentales pendant les essais, étant donné que ce comportement diffère de celui observé pour les autres matériaux étudiés où la variation est inférieure à $7 \%$. La diminution du module peut être liée aux relations utilisées pour la profondeur de contact et l'aire de contact, comme nous l'avons déjà mentionné. Malgré ces différences, le recouvrement entre les échelles valide l'hypothèse faite que le module de Young est constant quelle que soit la charge appliquée.

En revanche, il est reconnu que la dureté est une propriété qui varie avec la force appliquée. Il existe un effet de taille ou «ISE » (indentation size effect) dans chaque échelle, c'est-à-dire le plus souvent une dureté qui augmente lorsque la charge appliquée diminue. En nanoindentation, l'effet de taille est souvent expliqué par la théorie du «Strain Gradient Plasticity » (SGP) basée sur les dislocations géométriquement nécessaires pour accommoder la déformation plastique sous l'indenteur $[23,24]$. Dans le domaine de la microdureté, cet effet a été expliqué par différents phénomènes comme 

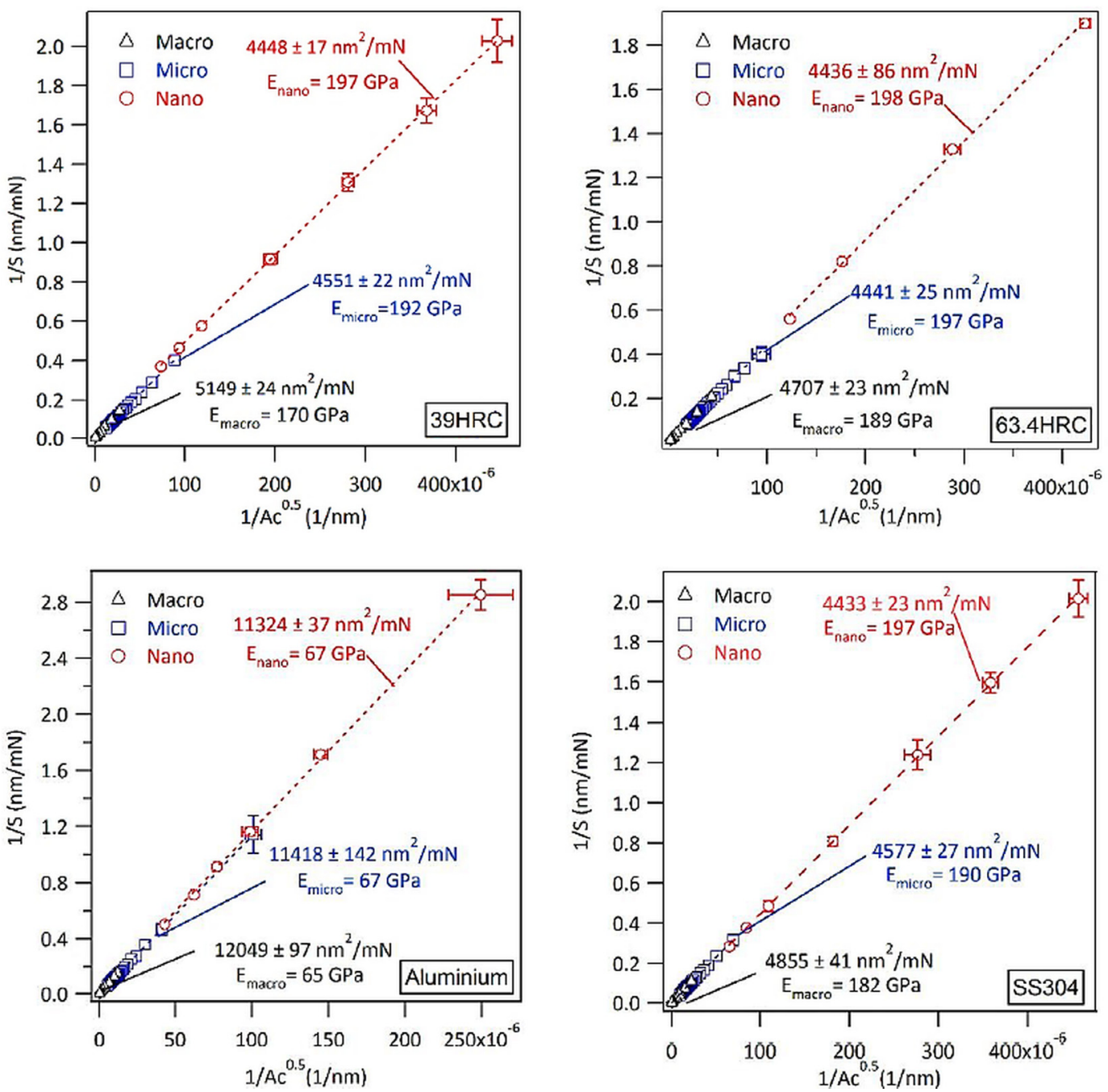

Fig. 5. Représentation de l'inverse de la raideur de contact en fonction de l'inverse de la racine carrée de l'aire de contact pour les essais effectués avec les trois instruments nano, micro et macro. Les résultats ont été déjà corrigés de la complaisance de la machine, $C_{\mathrm{f}}$. $E_{\text {nano }}, E_{\text {micro }}$ et $E_{\text {macro }}$, sont les modules de Young à chaque échelle. Mode de déformation : pile-up (39HRC, 63.4HRC et aluminium), sink-in (SS304).

Fig. 5. Representation of the inverse contact stiffness as a function of the inverse square root of the contact area for the tests performed with the three instruments nano, micro and macro. The results were corrected with the frame compliance, $C_{\mathrm{f}} . E_{\mathrm{nano}}$, $E_{\text {micro }}$ and $E_{\text {macro, }}$ are the Young's modulus of materials at each scale. Deformation mode: pile-up (39HRC, 63.4HRC and aluminum), sink-in (SS304).

l'écrouissage $[25,26]$, la charge nécessaire pour initier la déformation plastique [27], l'énergie d'activation pour la nucléation de dislocations [28], mais aussi considéré comme un artéfact lié l'estimation de l'aire de contact [23] ou au défaut de pointe $[29,30]$. En macroindentation la dureté est considérée habituellement comme constante, les variations pourraient être liées à l'estimation de l'aire de contact et à la formation du bourrelet. Toutefois, des études complémentaires doivent être menées pour mieux comprendre et expliquer les comportements observés à chaque échelle et pour chaque matériau.

Sur les Figures 6 et 7 , il y a différents éléments à mettre en évidence qui représentent le comportement général observé pour les quatre matériaux étudiés avec les trois instruments utilisés.
En nanoindentation, les essais en mode CSM et classiques sont comparables, les valeurs de la dureté et du module de Young ne varient pas significativement. A cette échelle il est très important de réaliser une calibration régulière de la pointe. Il faut vérifier toujours la complaisance de la machine $\left(C_{f}\right)$, laquelle peut avoir des valeurs non-nulles dues au montage de l'échantillon. Nous avons trouvé dans certains cas des valeurs de l'ordre de $10^{-2} \mathrm{~nm} / \mathrm{mN}$. Sans correction des déplacements et de la raideur, on peut obtenir une augmentation ou une diminution du module de Young avec la force; lequel redevient constant après correction de la complaisance, $C_{f}$. Par contre, la dureté est moins altérée par la prise en compte de la complaisance du bâti. 

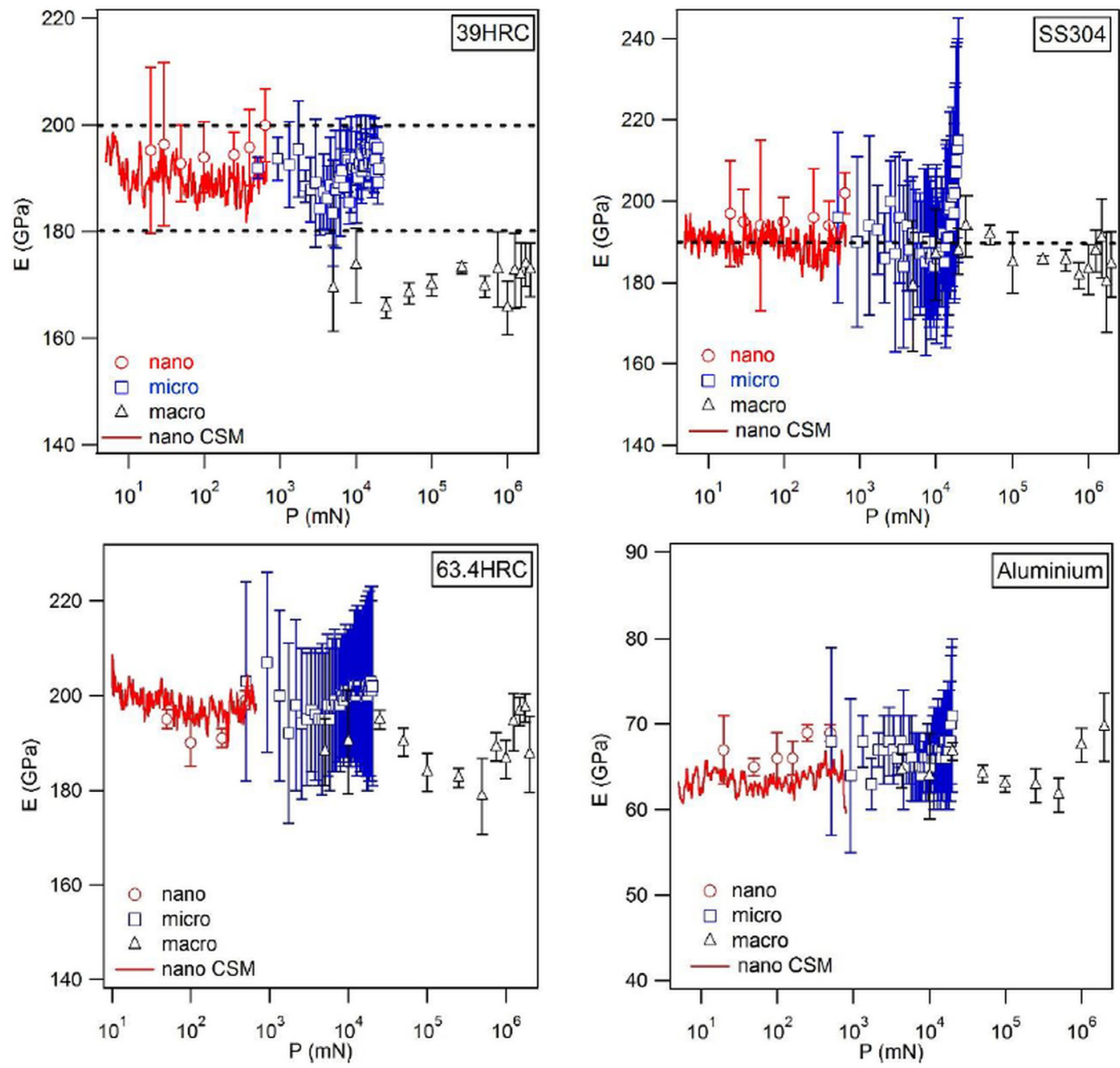

Fig. 6. Variation du module de Young en fonction de la force pour les matériaux étudiés, correspondant aux essais réalisés sur les trois machines nano, micro et macro indenteurs.

Fig. 6. Variation of Young's modulus as a function of load for the four studied materials and the three instruments nano, micro and macro indenters.

En microindentation, la correction de complaisance de la machine n'est volontairement pas prise en compte dans le logiciel du fait qu'elle varie selon la nature des matériaux, le montage de l'échantillon et les conditions d'essais. Dans ces conditions, la procédure de calibration de $C_{f}$ doit être effectuée à chaque série d'essais. Après cette correction, le module de Young est en général constant. Cependant, nous avons observé une augmentation du module vers les charges plus élevées, ce qui correspond au fait que la valeur de $C_{f}$ devient du même ordre de grandeur que celle de la complaisance totale mesurée. La précision sur la valeur de $C_{f}$ est donc déterminante pour estimer de manière correcte le module de Young et est par conséquent une source d'incertitude importante dans l'essai de microindentation. D'autre part, on peut aussi raisonnablement supposer qu'il existe une dépendance de la complaisance du bâti en fonction de la force appliquée, comme certains auteurs l'ont déjà proposé [31].

En macroindentation, même si la complaisance de la machine est prise en compte dans le logiciel après calibra- tion sur un matériau donné, sa valeur n'est pas forcément nulle lorsqu'on effectue un essai sur un autre matériau. Toutefois, les valeurs trouvées sont très faibles (de l'ordre de $10^{-4} \mathrm{~nm} / \mathrm{mN}$ ) et n'affectent les mesures effectuées que pour les charges supérieures à $100 \mathrm{~N}$, à partir desquelles le module de Young est modifié et corrigé. D'autre part, Ullner et al. [32], ont démontré pour une machine similaire que la complaisance varie avec la gamme de charge en macroindentation, cette approche peut donner des résultats plus précis, mais ce comportement n'est pas reproductible pour les essais effectués avec les différents échantillons. Là encore, des études complémentaires doivent être menées pour étudier la dépendance éventuelle de la complaisance avec la charge appliquée, que ce soit en micro ou en macroindentation.

Un élément très important pour l'appareil de macroindentation où l'échantillon est simplement posé, est la planéité de sa surface et son parallélisme par rapport au plan de la table $X Y$ de l'instrument. En effet, l'enregistrement de la courbe force-déplacement est très affecté 

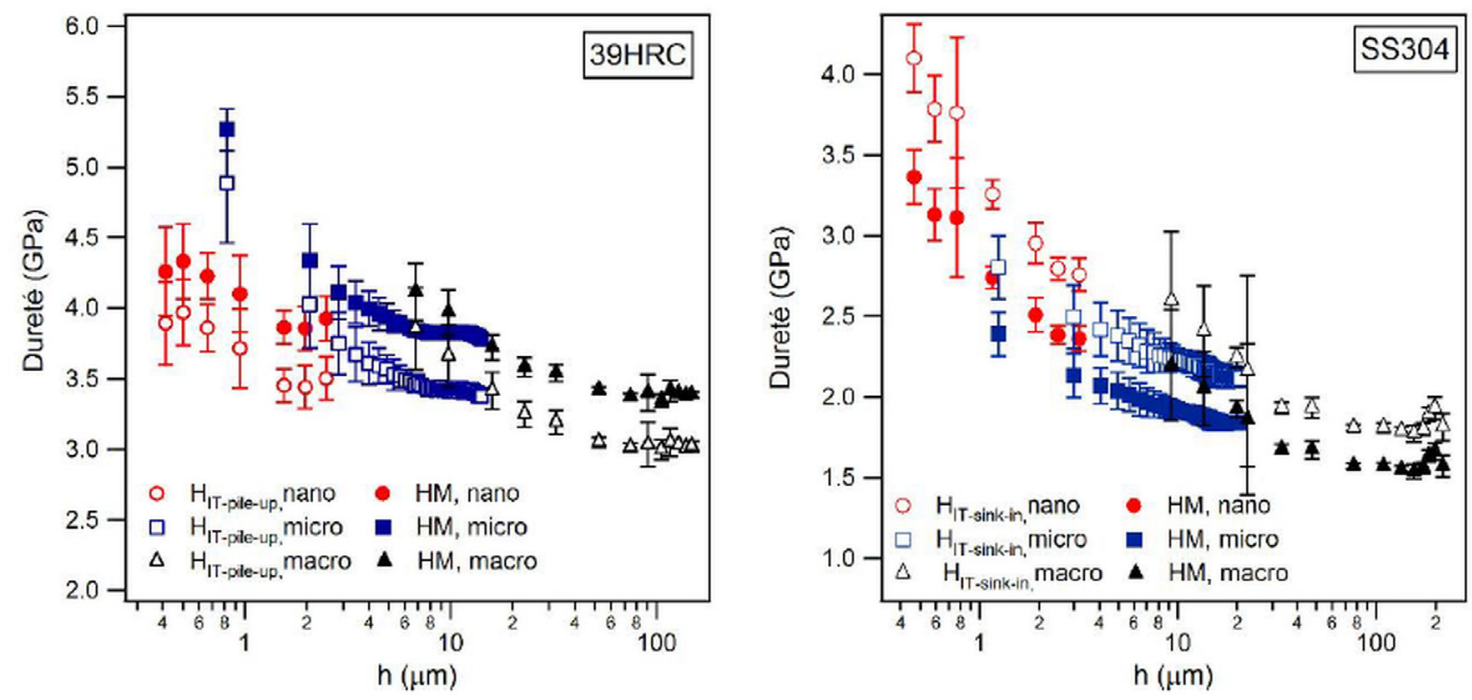

Fig. 7. Variation de la dureté en fonction du déplacement pour les matériaux 39HRC et SS304, correspondant aux essais réalisés avec les trois machines nano, micro et macro.

Fig. 7. Variation of hardness as a function of displacement for the materials 39HRC and SS304, corresponding to the tests performed with the three instruments nano, micro and macro.

lorsque l'échantillon bascule même très légèrement, c'est la raison pour laquelle nous utilisons de la pâte à modeler sur les bords de l'échantillon pour le maintenir en place.

\subsection{Comparaison avec des études inter-laboratoires précédentes (round-robin)}

Il existe plusieurs études de type round-robin qui ont présentés des comparaisons entre le module de Young obtenu avec différentes machines, comparaisons généralement réalisées en nanoindentation avec une méthodologie très rigoureuse $[31,33,34]$. La Figure $8 \mathrm{a}$ présente les variations du module de Young obtenues avec les trois machines utilisées dans notre étude et le résultat d'études comparatives trouvées dans la littérature.

L'étude nano (1) [34] fait la comparaison entre trois machines Fisherscope H100, Nanoindenter XP and UMIS2000 de différent laboratoires allemands. Les données sont traitées avec les logiciels de chaque instrument et aussi un logiciel supplémentaire (ASMEC Indent Analyser), les données de l'instrument UMIS-2000 sont analysées seulement avec ce logiciel. Tous les essais sont effectués à la même force, $500 \mathrm{mN}$, sur plus de 10 matériaux différents, céramiques, verres et métaux. Les écarts pour le module varient entre 7 et $35 \%$ par rapport aux valeurs mesurées par onde acoustique de surface (SAW) ou pris dans la littérature. L'analyse par le logiciel Indent Analyser diminue l'écart pour les données du Nanoindenter XP, mais les accentue pour celles du Fischerscope.

L'étude nano (2) [33] montre les variations trouvées entre douze participants internationaux dans l'étude d'un monocristal d'aluminium (100) Al avec deux gammes de charges de 0,1 à $5 \mathrm{mN}$ et de 10 à $100 \mathrm{mN}$. L'écart maximum entre les différents dispositifs expérimentaux dénommé comme coefficient de reproductibilité est $9 \%$ pour la gamme entre 1 et $5 \mathrm{mN}$ (où seulement six laboratoires ont participé), et $11 \%$ pour la gamme comprise entre 10 et $100 \mathrm{mN}$. Les écarts obtenus pour chaque participant appelé (coefficient de répétabilité) varient entre 1 à $17 \%$ en considérant les différentes charges, ces coefficients sont supérieurs pour la gamme des faibles charges.

L'étude nano (3) [31] compare les résultats de six machines, Hysitron UBI, Hysiton Triboscope, Micro Materials, Agilent G200, UNAT-ASMEC, et MTS XP). Ils utilisent les mêmes procédures pour la calibration de la complaisance du bâti et du défaut de pointe. Les données sont traitées avec le logiciel ASMEC Indent Analyser. Les essais sont réalisés pour des charges comprises entre 2 et $100 \mathrm{mN}$ avec trois indenteurs différents, cube corner, Berkovich et sphérique, sur deux types de matériaux, silice fondue $(\mathrm{FQ})$ et polycarbonate $(\mathrm{Pc})$. La Figure 8 présente les valeurs moyennes du module de Young pour l'indenteur Berkovich, la différence entre les machines change selon le matériau étudié, $3 \%$ pour FQ et $6 \%$ pour Pc, les écarts sont plus importants pour l'indenteur sphérique.

Finalement, nous pouvons considérer que les résultats de notre étude sont reproductibles aux différentes échelles en comparant les dispersions obtenues avec celles des différents round-robin. Les différences relatives estimées entre nano-micro, micro-macro et nano-macro ont des variations maximales de $12 \%$ entre-elles pour des charges comprises entre $5 \mathrm{mN}$ et $2 \mathrm{kN}$, soit une gamme de charge très supérieure à celles des études de la littérature, avec trois instruments très différents entre eux, et des matériaux industriels bien plus hétérogènes que ceux utilisés dans les études précédentes.

Dans cette perspective, les coefficients de variation ont été estimés aussi pour chaque machine par matériau, les valeurs ne dépassent pas $10 \%$ (Fig. 8b), les variations les plus importantes correspondent à la machine de 

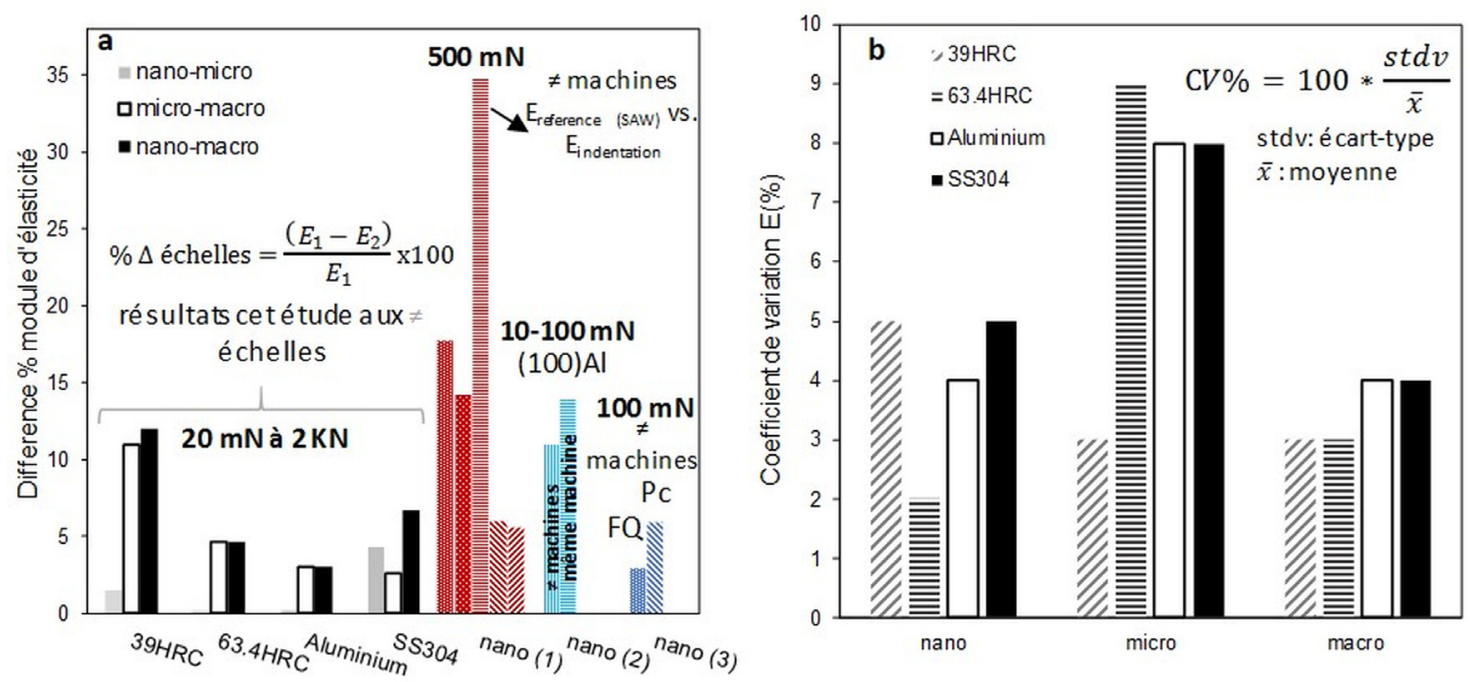

Fig. 8. (a) Variation du module de Young entre échelles et comparaison avec études précédents du type Round-Robin [31,33,34]. (b) Variation du module de Young par matériau dans les trois instruments. Le coefficient de variation est calculé en fonction de l'écart-type et la moyenne des résultats.

Fig. 8. (a) Variation of Young's modulus between scales and comparison with previous Round-Robin studies [31, 33, 34]. (b) Variation of Young's modulus for each material with the three instruments. The variation coefficient is computed considering the standard deviation and the average value of results.

microindentation, ceci est probablement lié au système de fixation. La norme ISO 14577 indique que le pourcentage d'erreur doit être inférieur à $5 \%$ pour une même charge. Cependant, nous avons considéré toute la gamme de charges étudiées pour chaque machine. Par conséquent, nous pouvons considérer les résultats comme cohérents, puisque les essais d'indentation sont affectés par de nombreuses sources d'incertitude possibles [21,35].

\section{Conclusions}

L'étude de l'indentation à différentes échelles de mesure peut donner une meilleure compréhension des phénomènes qui se produisent pendant l'essai d'indentation. Dans cette perspective, nous avons trouvé une continuité dans l'estimation du module de Young et des valeurs quasiment constantes pour chaque matériau, ces valeurs étant parfaitement compatibles avec celles issues de la littérature. L'analyse nécessite de considérer de manière rigoureuse la correction du défaut de pointe et la complaisance du bâti quelle que soit l'échelle de mesure et quel que soit la conception de l'instrument sur la mesure des déplacements. D'autre part, la dureté a montré aussi une continuité mais avec une tendance à la décroissance avec l'augmentation de la force appliquée, et l'effet de taille est visible aux trois échelles.

Les relations d'Oliver et Pharr et de Loubet et al. utilisées pour estimer les profondeurs de contact selon le mode de déformation, respectivement déflections des faces (SS304) et formation d'un bourrelet (aciers et alliage d'aluminium), représentent une bonne estimation pour les matériaux étudiés. Cependant, en macroindentation, ces relations doivent être vérifiées, du fait des écarts trouvés par rapport aux résultats obtenus en nano et en microindentation. Il est aussi important de mettre en évidence, que les facteurs de correction $\beta$ et $\gamma$ dans le module de Young, permettent une estimation plus proche des valeurs théoriques. Si nous utilisons uniquement le facteur $\gamma$, les résultats sont encore très cohérents et le module de Young augmente d'environ $4 \%$ par rapport aux valeurs montrées dans notre étude.

\section{Références}

[1] M.R. VanLandingham, J. Res. Natl. Inst. Stand. Technol. 108 (2003) 249-265

[2] J. Hay, Exp. Tech. 33 (2009) 66-72

[3] M. Dao, N. Chollacoop, K.J. Van Vliet, T.A. Venkatesh, S. Suresh, Acta Mater. 49 (2001) 3899-3918

[4] J.M. Antunes, J.V. Fernandes, L.F. Menezes, B.M. Chaparro, Acta Mater. 55 (2007) 69-81

[5] M. Mata, M. Anglada, J. Alcalá, J. Mater. Res. 17 (2002) 964-976

[6] F. Rickhey, K.P. Marimuthu, J.H. Lee, H. Lee, J.H. Hahn, Eng. Fract. Mech. 148 (2015) 134-144

[7] Y. Feng, T. Zhang, Acta Mech. Solida Sin. 28 (2015) 221-234

[8] E. Barthel, D. Chicot, J.P. Guin, E. Le Bourhis, G. Mauvoisin, L'indentation : un outil de caractérisation multi-échelle des matériaux, presented at the Commission thématique Indentation, SF2M Info, 2014, p. 13

[9] W.C. Oliver, G.M. Pharr, J. Mater. Res. 7 (1992) 15641583

[10] A.C. Fischer-Cripps, Surf. Coat. Technol. 200 (2006) 4153-4165

[11] D. Chicot, M. Yetna, E.S Puchi-Cabrera, A. Iost, M. Staia, G. Louis, G. Bouscarrat, R. Aumaitre, Thin Solid Films 558 (2014) 259-266 
[12] M. Troyon, L. Huang, Surf. Coat. Technol. 201 (2006) 1613-1619

[13] W.C. Oliver, G.M. Pharr, J. Mater. Res. 19 (2003) 3-20

[14] J.L. Loubet, M. Bauer, A. Tonck, S. Bec, B. GauthierManuel, Nanoindentation with a surface force apparatus, in Mechanical Properties and Deformation Behavior of Materials Having Ultra-Fine Microstructures, Springer, 1993, pp. 429-447

[15] G. Hochstetter, A. Jimenez, J.L. Loubet, J. Macromol. Sci. Part B 38 (1999) 681-692

[16] A. Bolshakov, G.M. Pharr, J. Mater. Res. 13 (1998) 1049-1058

[17] M.F. McGuire, Stainless Steels for Design Engineers. ASM International, 2008

[18] P.D.S. Rao, Strength of Materials : A Practical Approach. Universities Press, 1999

[19] J.E. Hatch, Aluminum: Properties and Physical Metallurgy. ASM International, 1984

[20] C. Ullner, Critical points in ISO 14577 part 2 and 3 considering the uncertainty in measurement, Proc HARDMEKO, 2004, pp. 11-12

[21] R. Cagliero, G. Barbato, G. Maizza, G. Genta, Int. J. Mech. Sci. 101-102 (2015) 161-169

[22] J. Thurn, D.J. Morris, R.F. Cook, J. Mater. Res. 17 (2002) 2679-2690
[23] W.D. Nix, H. Gao, J. Mech. Phys. Solids 46 (1997) 411425

[24] A.A. Elmustafa, D.S. Stone, J. Mech. Phys. Solids 51 (2003) 357-381

[25] H. O'Neill, The hardness of metals and its measurement. Chapman \& Hall, ltd., 1934

[26] B.W. Mott, Micro-indentation hardness testing. Butterworths Scientific Publications, 1956

[27] C. Hays, E.G. Kendall, Metallography 6 (1973) 275-282

[28] N. Gane, J.M. Cox, Philos. Mag. 22 (1970) 0881-0891

[29] A. Iost, R. Bigot, J. Mater. Sci. 31 (1996) 3573-3577

[30] D. Chicot, M.Y. N'Jock, F. Roudet, X. Decoopman, M.H. Staia, E.S. Puchi-Cabrera, Int. J. Mech. Sci. 105 (2016) 279-290

[31] M. Cabibbo, P. Ricci, R. Cecchini, Z. Rymuza, J. Sullivan, S. Dub, S. Cohen, Micron 43 (2012) 215-222

[32] C. Ullner, E. Reimann, H. Kohlhoff, A. Subaric-Leitis, Measurement 43 (2010) 216-222

[33] K. Herrmann, D.A. Lucca, M.J. Klopfstein, F. Menelao, Metrologia 47 (2010) S50-S58

[34] T. Chudoba, M. Griepentrog, Z. Für Met. 96 (2005) 1242-1246

[35] J. Menèík, M.V. Swain, J. Mater. Res. 10 (1995) 14911501 